\title{
Recognition-induced forgetting of schematically related pictures
}

\author{
Paul S. Scotti ${ }^{1}$ - Laura Janakiefski ${ }^{2} \cdot$ Ashleigh M. Maxcey $^{2}$
}

Published online: 2 January 2020

(C) The Psychonomic Society, Inc. 2020

\begin{abstract}
Recognition-induced forgetting is the category-specific forgetting of pictures that occurs when a subset of a category of pictures is recognized, leading to forgetting of the remaining pictures. We have previously shown that recognition-induced forgetting does not operate over categories created by temporal relationships, suggesting that this effect does not operate over episodic memory representations. Here we systematically tested whether schematically related categories of pictures are immune to recognitioninduced forgetting. We found that sufficiently weak schematically related memories are vulnerable to recognition-induced forgetting. These results offer an alternative interpretation for evidence that recognition-induced forgetting does not operate over episodic memory representations. Evidence that the strength of schematic grouping modulates forgetting supports a model of recognition-induced forgetting in which the key determinant of forgetting is moderate activation. This is the first demonstration that recognition-induced forgetting operates over perceptually distinct objects, demonstrating the ubiquity of such forgetting.
\end{abstract}

Keywords Long-term episodic memory $\cdot$ Human memory $\cdot$ Semantic memory

Humans automatically form categories consisting of similar objects in order to simplify the complexity of our visual environment (Croft \& Cruse, 2004; Taylor, 2003), increasing the efficiency of both perceptual processing and memory storage (Rakison \& Oakes, 2003). A category refers to a grouping of objects that necessarily share certain features (Corrigan, Eckman, \& Noonan, 1989), defined according to perceptual or associative concepts (Zentall, Wasserman, \& Urcuioli, 2014). Here we define perceptual concepts formed through inherent physical similarities as encompassing subordinatelevel categories (e.g., different types of cockatoos) and basic-level categories (e.g., different types of birds). Associative concepts are formed through experiences and encompass schemas, a single unit in memory that integrates prior knowledge of high-level relationships (e.g., a snake and a cactus are schematically related because they are typical of a desert schema; R. C. Anderson, 1984; Valcke, 2002), as well as superordinate-level categories, objects associated at a high

Ashleigh M. Maxcey

ammaxcey@gmail.com

1 Department of Psychology, Ohio State University, Columbus, OH, USA

2 Department of Psychology, Vanderbilt University, Nashville, TN, USA level of taxonomic organization that encompasses lower-level perceptual concepts (e.g., different types of animals). ${ }^{1}$ In other words, schematic knowledge involves the learned relationships between object categories, and taxonomic knowledge involves the hierarchical properties of a set of objects, with a superordinate category being at the top of the taxonomic hierarchy (Lewis, Poeppel, \& Murphy, 2015; Mirman, Landrigan, \& Britt, 2017). Despite the potentially distinct underlying neural mechanisms of perceptual and associative concepts (Mack \& Palmeri, 2011, 2015; Mareschal, Quinn, Lea, \& Lea, 2010), studies investigating forgetting have largely ignored these categorical distinctions, including our own studies of recognition-induced forgetting ${ }^{2}$ of pictures (but see Maxcey, Janakiefski, Megla, Smerdell, \& Stallkamp, 2019). Recognition-induced forgetting is the forgetting of objects held in memory following the recognition of a target memory from the same object category (Maxcey \& Woodman, 2014). ${ }^{3}$ We have employed the term object

\footnotetext{
${ }^{1}$ Although the development of concepts and category learning are both critical to interacting with the world (Sloutsky, 2010), they are beyond the scope of this work.

${ }^{2}$ See the General Discussion for a comparison between recognition- and retrieval-induced forgetting.

${ }^{3}$ The recognition-induced forgetting paradigm, by definition, presents additional, novel pictures in the old-new recognition judgment task. Our lab has demonstrated that forgetting is not parametrically manipulated by set size, suggesting that forgetting is not due to retroactive interference but is indeed the result of recognition (Maxcey, 2016).
} 
category in our previous work to broadly apply to both basicand subordinate-level categories. To generalize across these previous findings, we will use only the term basic-level category to describe the object relationships in the majority of our existing studies (Maxcey, 2016; Maxcey \& Bostic, 2015; Maxcey, Bostic, \& Maldonado, 2016; Maxcey, Janakiefski, et al., 2019; Maxcey \& Woodman, 2014; Rugo, Tamler, Woodman, \& Maxcey, 2017). Here we tested whether recognition-induced forgetting operates across schematically related ${ }^{4}$ memories.

Consider the following eyewitness example. If I witness a crime that involves a weapon, getaway car, and robber, and am then asked by police at the crime scene to identity the robber in a lineup, am I forgetting the weapon and the getaway car? The object relationships in this scenario differ from those in most studies of recognition-induced forgetting. In this eyewitness example, the objects are meaningfully related because they were encountered at the same time (i.e., are temporally grouped), and are associatively grouped by belonging to the schema of robbery. If they were grouped at the basic level, as in most recognition-induced forgetting studies, the objects would be drawn from the same basic-level category as the target (e.g., they would all be faces, like forgetting the face of the getaway car driver after identifying the face of the gunman). It is unknown whether recognition-induced forgetting only operates over such basic-level categories or also applies to schematically related objects.

Schematically related categories fundamentally differ from basic-level categories, because they consist of learned associative concepts composed of arbitrary stimuli related by accumulated experience, rather than by physical properties or inherent relationships (Lewis et al., 2015; Mirman et al., 2017; Zentall et al., 2014). For example, learning to categorize a tractor and a haystack as objects grouped under the schema farm occurs over time. Meanwhile, a bulldozer and a farm tractor can immediately be grouped on the basis of physical resemblance, under the basic-level category of tractor. Indeed, recent work from our lab (see Fig. 1) has suggested that recognitioninduced forgetting may not operate over temporally grouped objects (Maxcey, Glenn, \& Stansberry, 2018) or pictures grouped by schema (Maxcey, Janakiefski, et al., 2019, Exp. 3).

Our inability to find recognition-induced forgetting for temporally (Maxcey et al., 2018) or schematically (Maxcey, Janakiefski, et al., 2019) grouped objects led

\footnotetext{
${ }^{4}$ In a previous study (Maxcey, Janakiefski, et al., 2019) using this level of grouping, we employed the term superordinate rather than schematic. The types of relationships we tested here could also be referred to as superordinate at the very abstract level, or as thematically related if constrained to objects with different functional roles (Goldwater, Markman, \& Stilwell, 2011). Here, for the sake of consistency and precision, we use the term schematic to describe the associations among our stimuli.
}

us to conclude that recognition-induced forgetting does not operate over episodic memory (Maxcey et al., 2018). However, the following literature suggests that forgetting should operate over episodic memory (e.g., at the schematic level). First, prominent theories of forgetting that may account for recognition-induced forgetting are not constrained to semantic memory, including competitionbased accounts (Jakab \& Raaijmakers, 2009; LewisPeacock \& Norman, 2014; Norman, Newman, \& Detre, 2007; Raaijmakers \& Jakab, 2013a, 2013b), inhibition accounts (M. C. Anderson, 2003; Detre, Natarajan, Gershman, \& Norman, 2013), and context accounts (Jonker, Seli, \& MacLeod, 2013). Second, retrievalinduced forgetting, a seemingly closely related forgetting phenomenon, has been shown to operate over associative memories (Ciranni \& Shimamura, 1999; Gómez-Ariza, Fernandez, \& Bajo, 2012; Murayama, Miyatsu, Buchli, \& Storm, 2014) and is generally thought to operate over episodic memory (M. C. Anderson, 2003; Levy \& Anderson, 2002). Third, the potentially related cognitive phenomena of priming (Maxcey, McCann, \& Stallkamp, 2019), in which exposure to an object influences later processing of that and related objects, is also not restricted to semantic associations (Ochsner, Chiu, \& Schacter, 1994; Tulving \& Schacter, 1990). Here we asked whether recognition-induced forgetting operates over memory representations categorized at the schematic level. This issue warrants systematic examination because our previous conclusion that recognition-induced forgetting does not operate over episodic memory (Maxcey et al., 2018) is at odds with the work described above. If the mechanisms underlying recognition-induced forgetting do not operate over episodic memory representations, then schematically grouped objects should not be susceptible to recognitioninduced forgetting.

We modified the recognition-induced forgetting paradigm used by Maxcey, Janakiefski, et al. (2019), illustrated in Fig. 1, which presents objects grouped at the schematic level. In Maxcey, Janakiefski, et al. (2019), a text label above every study object explicitly informed the participant of the relevant schema (e.g., kitchen label above a refrigerator), thus serving as a shared recognition cue because the associated label always accompanied the objects in each phase of the experiment (i.e., study, practice, or test). In Experiment 1 (Fig. 2), we strengthened schematic associations by temporally grouping related objects during the study phase (as in Maxcey et al., 2018) but having only one item practiced at a time (as in Maxcey, Janakiefski, et al., 2019), to prevent interference during the practice phase. In Experiments 2 (see Fig. 4 below) and 3 , we further strengthened schematic associations by replacing the text labels with compelling background scenes that visually depicted the schema, as if the objects were encountered in the schematically consistent location. 
Study Phase

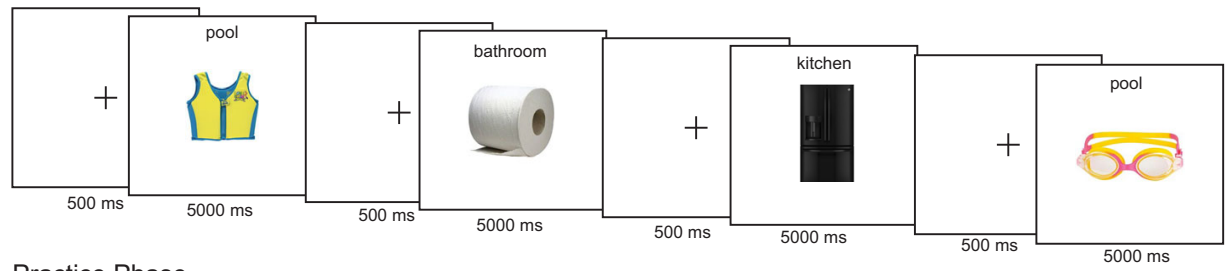

Practice Phase
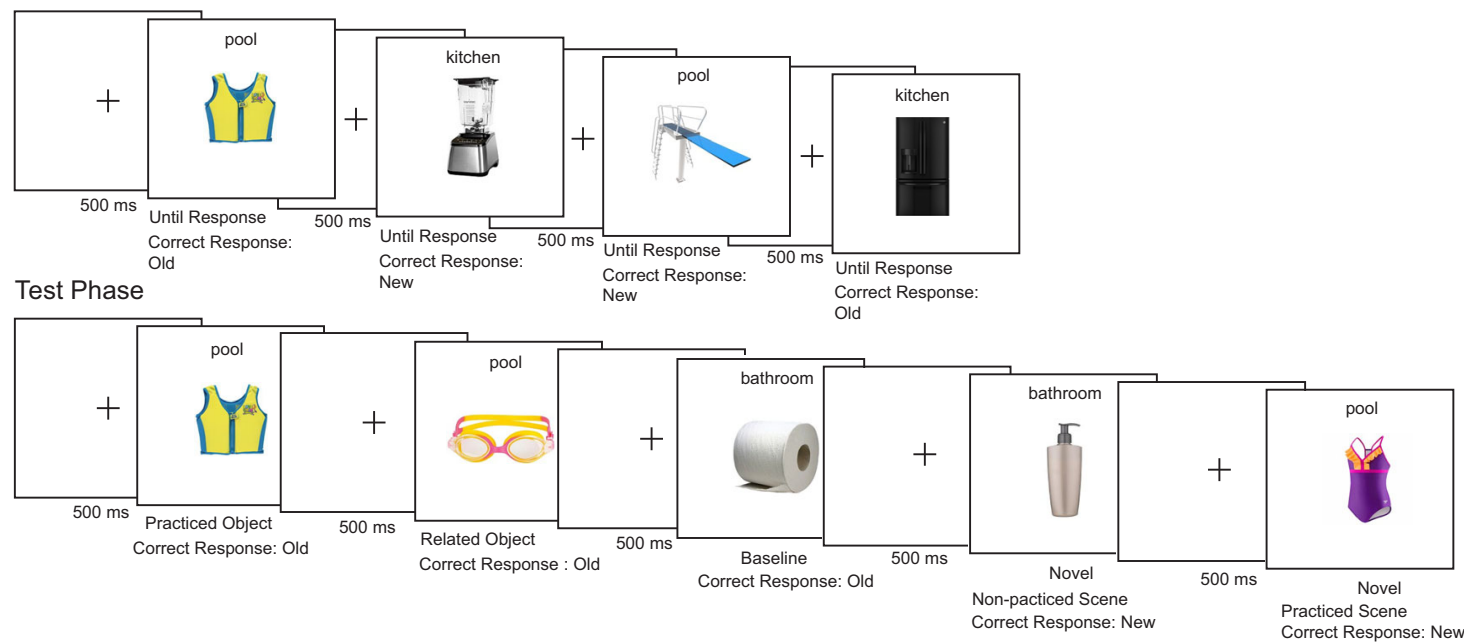

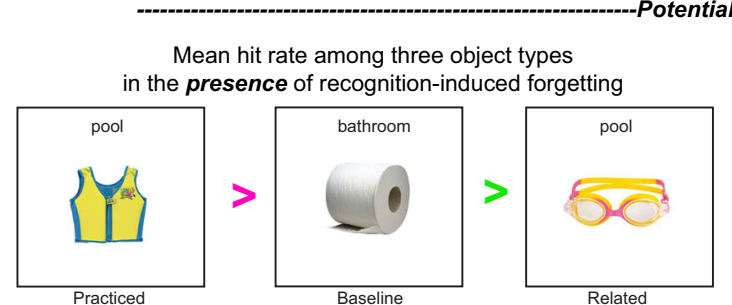

Fig. 1 Methods from Maxcey, Janakiefski, et al. (2019), Experiment 3. In the study phase, participants fixated on a central fixation point for $500 \mathrm{~ms}$, followed by presentation of each stimulus for $5,000 \mathrm{~ms}$, until all stimuli had been presented. Each object was accompanied by a scene name in which it could be found. The participants were instructed to remember each object for a later memory test. The study phase was followed by a 5 min visual distractor task. In the practice phase, half of the objects from half of the categories were again presented, along with an equal number of novel objects. Participants engaged in recognition practice by completing an old-new recognition task in response to each object. Each old object was practiced on two practice trials. The practice lures were objects

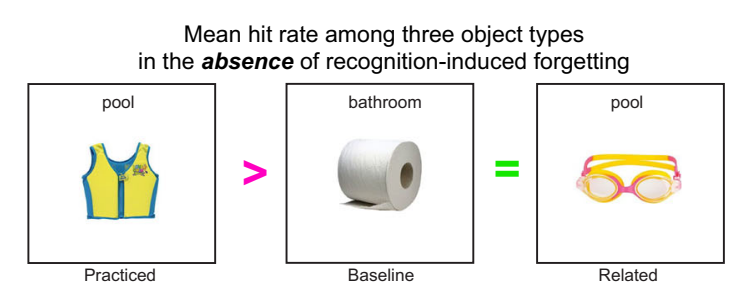

drawn from the same categories as the practiced objects. The second phase was followed by another 5-min visual distractor task. The test phase employed the same old-new recognition task as the second phase, but it included 36 old stimuli from the study phase (12 baseline, 12 practiced, and 12 related), as well as an equal number of novel test lures from the same categories. Hit rates from this test phase are presented in the data figures by object type. The bottom panel illustrates the expected patterns of results in the presence and absence of recognition-induced forgetting. Maxcey, Janakiefski, et al. (2019) found no recognition-induced forgetting (i.e., the pattern illustrated on the right).

\section{Study Phase}

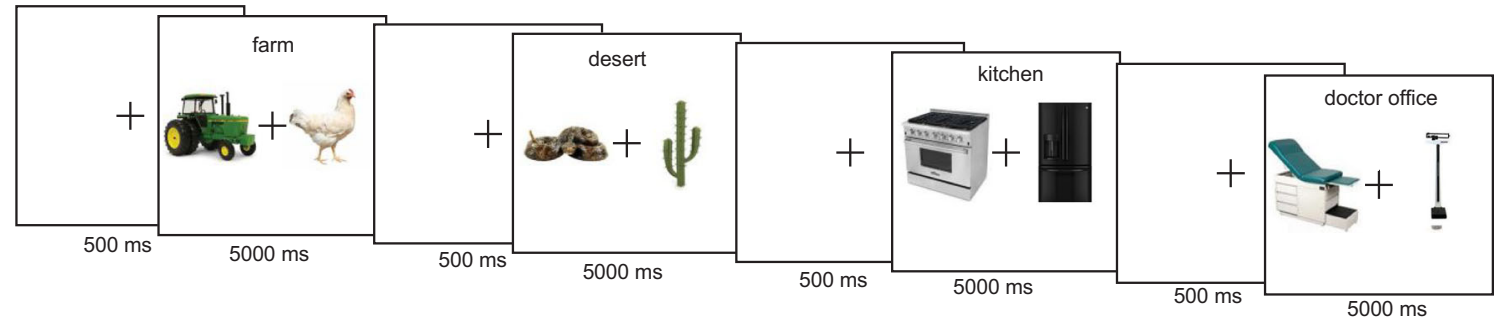

Fig. 2 Sample from the study phase of Experiment 1. Pairs of objects from the same schema were presented underneath a verbal label naming the associated schema. 


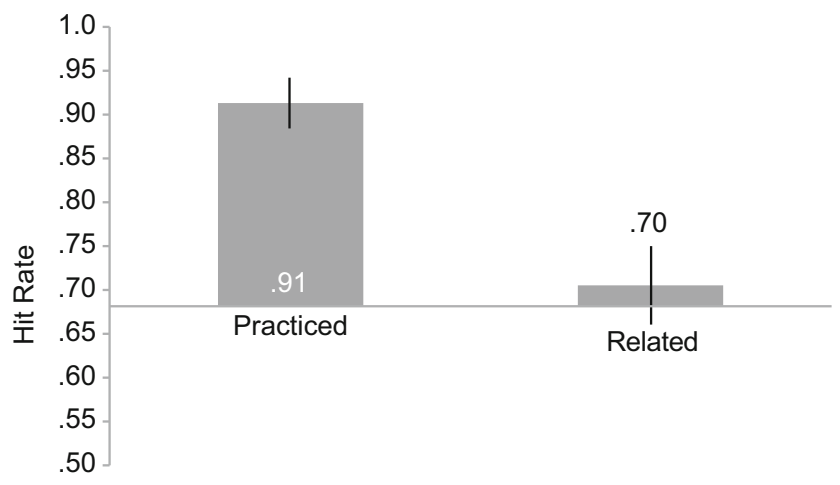

Fig. 3 Hit rates for old objects by object type in the test phase of Experiment 1 . In all data figures, the $x$-axis represents memory for baseline objects, and error bars represent $95 \%$ confidence intervals as described by Cousineau (2005) with Morey's correction applied (Morey, 2008).

\section{Experiment 1: Simultaneous study presentation with label}

To test whether objects categorized at the schematic level are susceptible to recognition-induced forgetting, we modified the standard recognition-induced forgetting paradigm. Objects were categorized at the schematic level (e.g., snake and cactus) instead of the basic level (e.g., two different cacti). To help participants relate objects at the intended schematic level, we presented a text label above objects that denoted the relevant theme (e.g., desert). The recognition practice phase consisted of an old-new recognition task with sequential object presentation, rather than the paired objects in previous studies (e.g., Maxcey et al., 2018; Maxcey \& Woodman, 2014), to prevent practice interference. ${ }^{5}$ Fourth, our sample size $(n=48)$ was considerably larger than those in most recognition-induced forgetting studies. All of these changes should, in theory, elicit robust recognition-induced forgetting effects by inducing stronger associations between the paired objects, reducing memory interference, and increasing statistical power.

We previously found that recognition-induced forgetting is not observed when objects are categorized at the schematic level (Maxcey, Janakiefski, et al., 2019, Exp. 3). However, an alternative explanation is that schematic grouping produces inherently weaker object associations. To boost the strength of object associations, we temporally grouped the objects, which has been shown to implicitly and automatically induce robust associations for attended objects (Fiser \& Aslin, 2002; Kirkham, Slemmer, \& Johnson, 2002; Olson \& Chun, 2001;

\footnotetext{
${ }^{5}$ Maxcey et al. (2018) may have obscured the evidence for recognitioninduced forgetting due to practice interference, because their recognition practice phase presented two objects to the left and right of fixation, and participants were instructed to report which object was old (i.e., which object had previously been presented in the study phase). This paired presentation could have interfered with memory for the studied object, such that the temporally grouped study objects could have been overwritten by the temporally grouped practice objects.
}

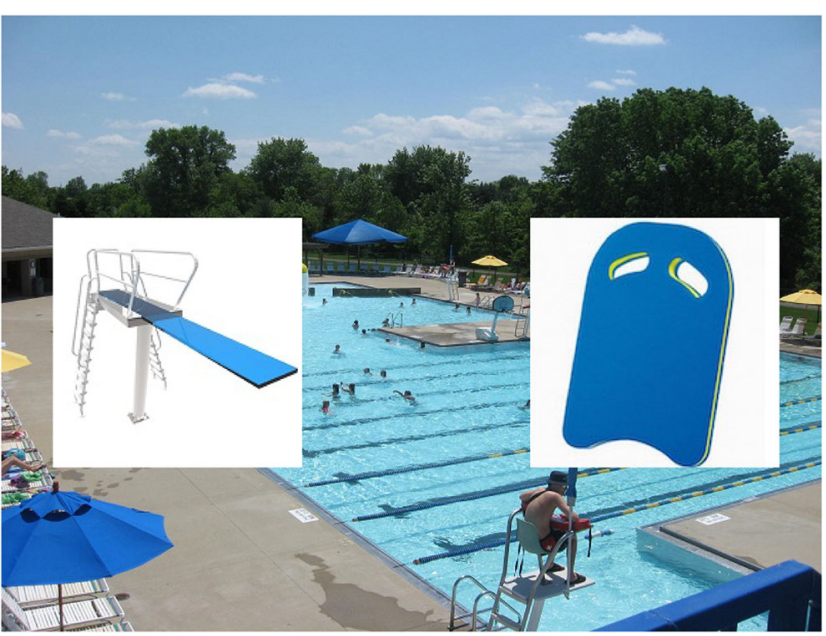

Fig. 4 Example of a study trial from Experiment 2. The studied objects were presented in front of a semantically consistent scene.

Turk-Browne, Jungé, \& Scholl, 2005). In Experiment 1, every study trial consisted of two objects that shared the same schema and were studied at the same point in time, with their corresponding schema label displayed above (Fig. 2).

\section{Method}

Participants The participants were 48 Ohio State University undergraduates (mean age of 19.4 years; 28 female, 20 male) who completed the experiment in exchange for course credit.

Power analyses were performed using $\mathrm{G}^{*}$ Power (Faul, Erdfelder, Lang, \& Buchner, 2007), to determine the necessary sample size for recognition-induced forgetting studies, using the smallest effect size measured in the original recognition-induced forgetting study, which is typical of the literature on recognition-induced forgetting $\left(d_{\mathrm{z}}=1.376\right.$; Maxcey \& Woodman, 2014, Exp. 1). We estimated that a sample size of 12 participants per experiment would be necessary in order to observe recognition-induced forgetting effects with $99 \%$ power, given a .05 criterion of significance. We anticipated null results (i.e., no recognition-induced forgetting as measured by baseline minus related), so we chose a sample size of 48-50 participants in each experiment, to ensure adequate statistical power.

Stimuli The experiment was programmed using E-Prime (Schneider, Eschman, \& Zuccolotto, 2012). Participants were seated approximately $80 \mathrm{~cm}$ from the monitor, and the stimuli subtended approximately $4.6 \mathrm{deg}$ of visual angle. The object categories in each experiment included 120 objects belonging to 12 categories (counterbalanced across subjects) drawn from a larger set of 24 categories. ${ }^{6}$

\footnotetext{
${ }^{6}$ The full stimulus set is on the OSF site: https://osf.io/5s $96 \mathrm{y} /$ ?view only= e1801f074078415f90e0e3e536b33b5f.
} 
Procedure The general procedure of recognition-induced forgetting involves a study phase, a practice phase, and a test phase. Studied objects can belong to one of three object types: practiced objects, shown during the study and practice phases; related objects, or the nonpracticed objects from the pairs to which the practiced objects belonged; and baseline objects, or objects from the study-phase pairs that were never practiced. During the practice and test phases, participants perform an old-new recognition judgment of whether they have seen a given object before (we refer to the new objects as lures). Recognition-induced forgetting is demonstrated if memory performance is poorer for related objects than for baseline objects during the test phase. Our modification to this general procedure is illustrated in Fig. 2: In the study phase, each trial consisted of two objects from the same schema presented simultaneously below the name of the relevant schematic category.

Each of the 24 trials lasted for $5 \mathrm{~s}$, interleaved with a 500ms fixation cross. Participants were instructed to remember the objects for a later memory test. The study phase was followed by a 5-min delay, during which the participants engaged in the attentionally demanding visual task of searching for Waldo in Where's Waldo books.

Next, in the recognition practice phase, half of the objects from half of the studied categories (i.e., the practiced objects) were presented sequentially below a schematic category label, and participants were instructed to make an old-new recognition judgment, indicating whether or not they had seen the object before. The practiced objects were each practiced twice during this phase, whereas each lure (drawn from the same category as the practiced objects) was seen only once. Hence, we needed twice as many unique lures as practiced objects, requiring two new objects from each of the 12 schematic categories included in the practice phase. The practice phase included 48 total trials ( 24 old, 24 new).

Another 5-min delay activity intervened between the practice and test phases. The task during the test phase was the same as that during the practice phase. In this final old-new recognition task, 48 new objects were presented sequentially, evenly divided from each schematic category, along with all 48 old objects from the 24 studied pairs (12 related objects, 12 practiced objects, and 24 baseline objects), totaling 96 test phase trials.

Data analysis The primary dependent variable for recognition memory is hit rate across the three main object types (practiced, related, baseline). We report both the practice benefit (practice - baseline) and recognition-induced forgetting (baseline - related), although the latter is the measurement of interest. In the data figures, the magnitude of the practice benefit is illustrated by the size of the left bar (where the baseline is the $x$-axis), and the degree of recognition-induced forgetting is illustrated by the size of the right bar. To provide converging evidence for our hit rate analyses, in the footnotes beneath the critical comparisons we also report the discrimination measure
$P r$, which is hits - false alarms, and the associated bias measure $\mathrm{Br}^{7}$ (Feenan \& Snodgrass, 1990). Preplanned $t$ tests are accompanied by JZS Bayes factors to quantify support for the null or alternative hypothesis (Rouder, Speckman, Sun, Morey, \& Iverson, 2009). The JZS Bayes factor tells us the relative probability of the data under one model (the null hypothesis) as compared to another model (the alternative hypothesis). For example, $\mathrm{JZS}_{\mathrm{ALT}}=3$ means that the data are three times more probable under one model (the alternative hypothesis) than under the other model (the null). Significant $t$ tests are accompanied by Cohen's $d$.

\section{Results and discussion}

Replicating Maxcey, Janakiefski, et al. (2019), a repeated measures analysis of variance (ANOVA) comparing the means for the baseline, related, and practiced objects $[F(2$, 94) $\left.=50.65, p<.001, \eta^{2}=.52\right]$ indicated a reliable difference among the three object types (Fig. 3). The mean hit rate was significantly better for practiced objects $(.91, S D=.14)$ than for baseline objects $(.68, S D=.21), t(47)=8.33, p<.001, d=$ $1.31, \mathrm{JZS}_{\mathrm{ALT}}=121,808,230$, demonstrating a practice effect (Fig 3, left bar). Consistent with our prediction that there would be no recognition-induced forgetting, related memories $(.70, S D=.21)$ were not significantly different from baseline (.68), $t(47)=0.98, p=.331, \mathrm{JZS}_{\mathrm{NULL}}=6.05$, Fig. 3 , right bar. ${ }^{8}$ The absence of recognition-induced forgetting here replicates the results of Maxcey et al. (2018) while controlling for practice interference.

\section{Experiment 2: Simultaneous study presentation with a background scene}

One lingering question from Experiment 1 was whether participants were actually using the provided text labels to categorize the related objects at the intended schematic level. To encourage participants to group the objects as intended, in Experiment 2 the study trials (Fig. 4) consisted of two objects displayed on a schematically consistent background scene (replacing the text label). We also added a fourth phase, following the test phase, in which memory for the object pairs was tested, to ensure that participants had explicit knowledge of our intended schematic-level categories and successfully remembered the pairs.

\footnotetext{
${ }^{7}$ When calculating $B r, \operatorname{Pr}$ values of 1 were changed to .99 . For more information about $P r$, see Ian Neath's useful website at https://memory.psych.mun. $\mathrm{ca} /$ models/recognition/index.shtml.

${ }^{8}$ The baseline $\operatorname{Pr}(.60, S D=.24)$ was statistically indistinguishable from the related $\operatorname{Pr}(.63, S D=.24), t(47)=0.982, p=.331, \mathrm{JZS}_{\mathrm{NULL}}=4.05$, with conservative biases for both baseline $(B r=.21, S D=.17)$ and related $(B r=$ $.26, S D=.26$ ).
} 


\section{Method}

Participants The participants were 50 new Ohio State University undergraduates (mean age of 19.6 years; 19 female, 31 male), who completed the experiment in exchange for course credit.

Stimuli and procedure The stimuli and procedure were identical to those in Experiment 1, with the following exceptions. The experiment was programmed using HTML, CSS, and JavaScript. Each object subtended approximately $3.78 \mathrm{deg}$ of visual angle, and each background scene subtended approximately $11.33 \mathrm{deg}$ of visual angle. The schematic category label was replaced with a background scene consistent with the schematic category (see Fig. 4). A fourth phase was also included, in which memory for the object pairs was tested, consisting of two objects presented on the screen. Twelve old trials consisted of a randomly selected study trial, and 12 new trials consisted of two objects: one an old object drawn from a practiced category, and one a new object drawn from the same category as the object it replaced. The attentionally demanding distractor task consisted of $5 \mathrm{~min}$ of change detection trials (e.g., Luck \& Vogel, 1997). The experiment is available to try online. ${ }^{9}$

\section{Results and discussion}

Replicating Experiment 1 and Maxcey, Janakiefski, et al. (2019, Exp. 3), a repeated measures ANOVA comparing the mean hit rates for the baseline, related, and practiced objects $\left[F(2,98)=17.707, p<.001, \eta^{2}=.265\right]$ indicated a reliable difference among the three object types (Fig. 5). The mean hit rate was significantly better for practiced objects $(.95, S D=$ $.07)$ than for baseline objects $(.87, S D=.13), t(49)=4.649, p$ $<.001, d=0.70, \mathrm{JZS}_{\mathrm{ALT}}=805.85$, demonstrating a reliable practice benefit (Fig. 5, left bar). Again, related memories $(.85, S D=.14)$ were not significantly different from baseline $(.87), t(49)=1.135, p=.262, \mathrm{JZS}_{\mathrm{NULL}}=3.55,{ }^{10}$ revealing the absence of recognition-induced forgetting (Fig. 5, right bar). Memory for the old pairs presented in the fourth phase averaged an accuracy of $.94(S D=.08)$, significantly better than chance, $t(49)=40.288, p<.001, d=5.70, \mathrm{JZS}_{\mathrm{ALT}}=$ $5.25 \times 10^{35}$, ruling out the possibility that recognition-induced forgetting was not observed simply because of a failure to remember the study-phase pairs throughout the experiment. The absence of recognition-induced forgetting here replicates the results of Experiment 1 and of Maxcey, Janakiefski, et al. (2019) and suggests that insufficiently compelling temporal

\footnotetext{
9 https://maxceylab.github.io/expts/compelling_scenes/5.html

${ }^{10}$ The baseline $\operatorname{Pr}(.83, S D=.15)$ was statistically indistinguishable from the related $\operatorname{Pr}(.81, S D=.15), t(49)=1.135, p=.262, \mathrm{JZS}_{\mathrm{NULL}}=3.55$, with conservative biases for both baseline $(B r=.33, S D=.38)$ and related $(B r=$ $.24, S D=.29)$.
}

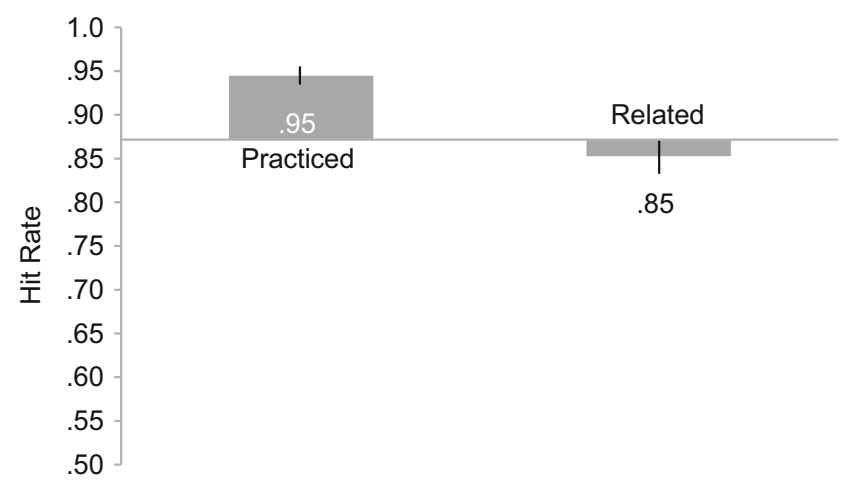

Fig. 5 Mean hit rates by object type in the test phase of Experiment 2.

grouping cues cannot explain our lack of recognitioninduced forgetting effects.

\section{Experiment 3: Replicate Experiment 2 with half the exposure duration at study}

The purpose of Experiment 2 was to encourage participants to notice the schematic relationship between the two objects. In Experiment 2, one may argue that recognition-induced forgetting was not observed due to ceiling effects, since the baseline performance went up 19\% from Experiment 1 (68\%) to Experiment 2 (87\%). The purpose of Experiment 3 was to lower the performance using the same task as in Experiment 2. To accomplish this, we cut the exposure duration of study phase objects in half in Experiment 3, from 5 to $2.5 \mathrm{~s}$. This enabled us to ask whether forgetting occurred for schematically related objects at a different range of memorability, when the task was harder and memory for the objects was lower.

\section{Method}

Participants The participants were 50 new Vanderbilt University undergraduates (mean age of 18.7 years; 43 female, 7 male), who completed the experiment in exchange for course credit.

Stimuli and procedure The stimuli and procedure were identical to those in Experiment 2, except that the exposure duration during the study phase was decreased from 5 to $2.5 \mathrm{~s}$. The experiment was approved by the Vanderbilt University Institutional Review Board and is available to try online. ${ }^{11}$

\section{Results and discussion}

A repeated measures ANOVA comparing the mean hit rates for baseline, related, and practiced objects $[F(2,98)=29.299$, $\left.p<.001, \eta^{2}=.374\right]$ indicated a reliable difference among the

\footnotetext{
${ }^{11} \mathrm{https}: / /$ maxceylab.github.io/expts/compelling_scenes/2point5.html
} 
three object types (Fig. 6). The mean hit rate was significantly better for practiced objects $(.92, S D=.08)$ than for baseline objects $(.81, S D=.18), t(49)=5.310, p<.001, d=0.80$, $\mathrm{JZS}_{\mathrm{ALT}}=6,585$, demonstrating a reliable practice effect (Fig. 6, left bar). Unlike in the previous experiments, and contrary to our prediction, related memories $(.77, S D=.18)$ were significantly lower than baseline $(.81), t(49)=2.571, p=$ $.013, d=0.27, \mathrm{JZS}_{\mathrm{ALT}}=2.95,{ }^{12}$ showing reliable recognitioninduced forgetting (Fig. 6, right bar). The recognition-induced forgetting here was likely due to the increased task difficulty relative to Experiment 2, since the methods were exactly the same between these two experiments, except for the study phase exposure duration. Indeed, it appears that memory for schematically grouped pairs was more fragile in Experiment 3 than in Experiment 2, measured by accuracy in the fourth phase, in which memory for the pairs was tested: Participants averaged an accuracy of $.87(S D=.14)$ in Experiment 3, which was significantly lower than their performance in the fourth phase of Experiment $2(.94, S D=.08)$, $t(49)=2.805, p=.007, d=0.61, \mathrm{JZS}_{\mathrm{ALT}}=4.99$.

\section{General discussion}

Here we found recognition-induced forgetting for perceptually distinct memory representations grouped by schema (Exp. 3 ), demonstrating that the mechanisms that underlie recognition-induced forgetting can operate over episodic memory. This is the first demonstration that this forgetting effect can be elicited by memories grouped at a representational level beyond the basic level employed in other studies of this effect (Maxcey, 2016; Maxcey \& Bostic, 2015; Maxcey et al., 2016; Maxcey, Janakiefski, et al., 2019; Maxcey \& Woodman, 2014; Rugo et al., 2017), contrary to our previous evidence that recognition-induced forgetting does not operate over higher-order groupings (Maxcey et al., 2018; Maxcey, Janakiefski, et al., 2019). When schematic relationships were relatively weak (Exp. 1) or strong (Exp. 2 ), recognition-induced forgetting was not observed. These results are consistent with models of forgetting that argue that the key determinant in forgetting is moderate activation (Detre et al., 2013; Lewis-Peacock \& Norman, 2014; Norman et al., 2007).

We note that there is some ambiguity as to whether schematically related memories are episodic or semantic. On the one hand, schematically related memories are created on the basis of accumulated experience learning that certain objects often co-occur together, which fits the definition of episodic memory (i.e., information about temporally related events or

\footnotetext{
${ }^{12}$ Baseline $\operatorname{Pr}(.75, S D=.21)$ was reliably higher than related $\operatorname{Pr}(.71, S D=$ .21) $\left[t(49)=2.559, p=.014, \mathrm{JZS}_{\mathrm{ALT}}=2.88\right)$, and conservative biases for both baseline $(B r=.25, S D=.22)$ and related $(B r=.24, S D=.27)$.
}

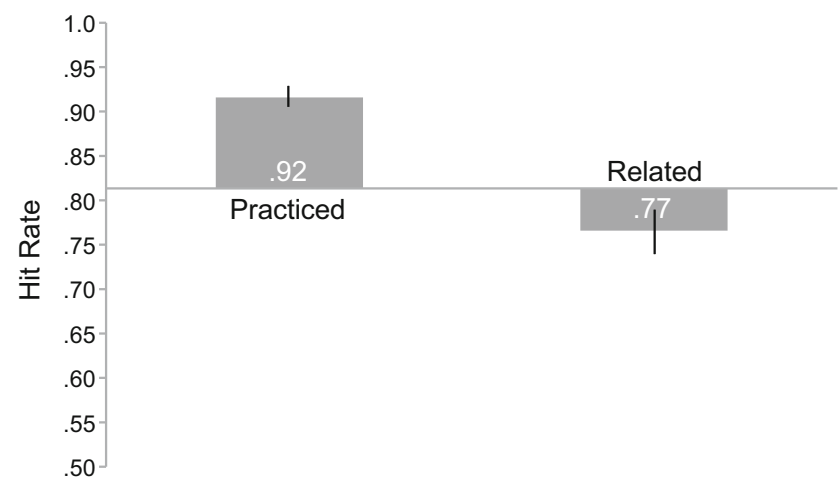

Fig. 6 Mean hit rates by object type in the test phase of Experiment 3.

relationships; Tulving, 1972). On the other hand, schematically related memories may also be semantically related (e.g., you are unlikely to find many objects in your kitchen that are semantically inconsistent with a kitchen schema). It is therefore still unclear whether recognition-induced forgetting can occur in the absence of any semantic relatedness. Indeed, this ambiguity has played a role in the argument that episodic memory and semantic memory may not be separate systems (McKoon, Ratcliff, \& Dell, 1986). We argue that the results of Experiment 3 demonstrate that recognition-induced forgetting can operate over episodic memory (in addition to semantic memory), because episodic association was one of three factors, in addition to moderate memory strength and semantic relatedness, shown to be important to eliciting recognitioninduced forgetting in perceptually distinct objects. Only by helping participants strengthen the episodic associations between objects via a compelling schema was the forgetting effect observed, demonstrating the involvement of episodic memory mechanisms in recognition-induced forgetting, and more generally supporting the idea that episodic and semantic memory systems may not be independent.

What is the relationship between recognition- and retrievalinduced forgetting? Recognition-induced forgetting is similar to retrieval-induced forgetting (M. C. Anderson, Bjork, \& Bjork, 1994). Their distinguishing feature is the task that induces forgetting: One is a recognition task, and one a recall task. Both these tasks are retrieval tasks according to the classic model of memory, in which the three stages of memory are encoding $\rightarrow$ storage $\rightarrow$ retrieval (Melton, 1963). Retrievalinduced forgetting has typically been interpreted as involving episodic retrieval (M. C. Anderson, 2003; Levy \& Anderson, 2002), employing stimuli grouped by schema (Storm \& Levy, 2012), operating over semantically unrelated associations (Ciranni \& Shimamura, 1999; Gómez-Ariza et al., 2012), and even transcending languages (Levy, McVeigh, Marful, \& Anderson, 2007).

The previous studies demonstrating recognition-induced forgetting used hierarchically lower category levels of objects, which may have been confounded by perceptual similarity. This means that the objects shared similar perceptual and 
semantic information (e.g., two feathers necessarily contain similar physical properties). Here we demonstrated that when the perceptual similarity of related objects was weakened, with measures being taken to strengthen the semantic similarity of schematic relationships, recognition-induced forgetting effects can be observed. These results inform models of forgetting by demonstrating that recognition-induced forgetting can operate over objects that lack perceptual similarities (see also Hong, Maxcey, \& Leber, 2018; Hong, Scotti, Maxcey, \& Leber, 2019), a finding that establishes an increased generalizability of recognition-induced forgetting and suggests underlying mechanisms similar to those in retrieval-induced forgetting.

\section{References}

Anderson, M. C. (2003). Rethinking interference theory: Executive control and the mechanisms of forgetting. Journal of Memory and Language, 49, 415-445. https://doi.org/10.1016/j.jml.2003.08.006

Anderson, M. C., Bjork, R. A., \& Bjork, E. L. (1994). Remembering can cause forgetting: Retrieval dynamics in long-term memory. Journal of Experimental Psychology: Learning, Memory, and Cognition, 20, 1063-1087. https://doi.org/10.1037//0278-7393.20.5.1063

Anderson, R. C. (1984). Role of the reader's schema in comprehension, learning, and memory. In R. C. Anderson, J. Osborn, \& R. J. Tierney (Eds.), Learning to read in American schools: Basal readers and content texts (pp. 243-257). Hillsdale, NJ: Erlbaum.

Ciranni, M. A., \& Shimamura, A. P. (1999). Retrieval-induced forgetting in episodic memory. Journal of Experimental Psychology: Learning, Memory, and Cognition, 25, 1403-1414. https://doi.org/ 10.1037/0278-7393.25.6.1403

Corrigan, R., Eckman, F. R., \& Noonan, M. P. (1989). Linguistic categorization (Vol. 61). Amsterdam, The Netherlands, NY: John Benjamins.

Cousineau, D. (2005). Confidence intervals in within-subject designs: A simpler solution to Loftus and Masson's method. Tutorial in Quantitative Methods for Psychology, 1, 42-45. https://doi.org/10. 20982/tqmp.01.1.p042

Croft, W., \& Cruse, D. A. (2004). Cognitive linguistics. New York, NY: Cambridge University Press.

Detre, G. J., Natarajan, A., Gershman, S. J., \& Norman, K. A. (2013). Moderate levels of activation lead to forgetting in the think/no-think paradigm. Neuropsychologia, 51, 2371-2388. https://doi.org/10. 1016/j.neuropsychologia.2013.02.017

Faul, F., Erdfelder, E., Lang, A.-G., \& Buchner, A. (2007). G*Power 3: A flexible statistical power analysis for the social, behavioral, and biomedical sciences. Behavior Research Methods, 39, 175-191. https:// doi.org/10.3758/BF03193146

Feenan, K., \& Snodgrass, J. G. (1990). The effect of context on discrimination and bias in recognition memory for pictures and words. Memory \& Cognition, 18, 515-527.

Fiser, J., \& Aslin, R. N. (2002). Statistical learning of higher-order temporal structure from visual shape sequences. Journal of Experimental Psychology: Learning, Memory, and Cognition, 28, 458-467. https://doi.org/10.1037/0278-7393.28.3.458

Goldwater, M. B., Markman, A. B., \& Stilwell, C. H. (2011). The empirical case for role-governed categories. Cognition, 118, 359-376.

Gómez-Ariza, C. J., Fernandez, A., \& Bajo, M. T. (2012). Incidental retrieval-induced forgetting of location information. Psychonomic
Bulletin \& Review, 19, 483-489. https://doi.org/10.3758/PBR.19.3. 483

Hong, Y., Maxcey, A. M., \& Leber, A. B. (2018, November). Recognition-induced forgetting of temporally related visual episodic memories. Poster presented at the Annual Meeting of the Psychonomic Society, New Orleans, LA.

Hong, Y., Scotti, P. S., Maxcey, A. M., \& Leber, A. B. (2019), Recognition-induced forgetting of temporally related visual longterm memories following training. Retrieved from https://osf.io/ ufkse. Accessed 27 Jul 2019.

Jakab, E., \& Raaijmakers, J. G. W. (2009). The role of item strength in retrieval-induced forgetting. Journal of Experimental Psychology: Learning, Memory, and Cognition, 35, 607-617. https://doi.org/10. 1037/a0015264

Jonker, T. R., Seli, P., \& MacLeod, C. M. (2013). Putting retrievalinduced forgetting in context: An inhibition-free, context-based account. Psychological Review, 120, 852-872. https://doi.org/10. 1037/a0034246

Kirkham, N. Z., Slemmer, J. A., \& Johnson, S. P. (2002). Visual statistical learning in infancy: Evidence for a domain general learning mechanism. Cognition, 83, B35-B42. https://doi.org/10.1016/s00100277(02)00004-5

Levy, B. J., \& Anderson, M. C. (2002). Inhibitory processes and the control of memory retrieval. Trends in Cognitive Sciences, 6, 299 305. https://doi.org/10.1016/S1364-6613(02)01923-X

Levy, B. J., McVeigh, N. D., Marful, A., \& Anderson, M. C. (2007). Inhibiting your native language: The role of retrieval-induced forgetting during second-language acquisition. Psychological Science, 18, 29-34. https://doi.org/10.1111/j.1467-9280.2007.01844.x

Lewis, G. A., Poeppel, D., \& Murphy, G. L. (2015). The neural bases of taxonomic and thematic conceptual relations: An MEG study. Neuropsychologia, 68, 176-189.

Lewis-Peacock, J. A., \& Norman, K. A. (2014). Competition between items in working memory leads to forgetting. Nature Communications, 5, 5768. https://doi.org/10.1038/ncomms6768

Luck, S. J., \& Vogel, E. K. (1997). The capacity of visual working memory for features and conjunctions. Nature, 390, 279-281. https://doi. org $/ 10.1038 / 36846$

Mack, M. L., \& Palmeri, T. J. (2011). The timing of visual object categorization. Frontiers in Psychology, 2, 165. https://doi.org/10.3389/ fpsyg.2011.00165

Mack, M. L., \& Palmeri, T. J. (2015). The dynamics of categorization: Unraveling rapid categorization. Journal of Experimental Psychology: General, 144, 551-569. https://doi.org/10.1037/ a0039184

Mareschal, D., Quinn, P. C., Lea, S. E., \& Lea, S. (2010). The making of human concepts. New York, NY: Oxford University Press.

Maxcey, A. M. (2016). Recognition-induced forgetting is not due to category-based set size. Attention, Perception, \& Psychophysics, 78, 187-197. https://doi.org/10.3758/s13414-015-1007-1

Maxcey, A. M., \& Bostic, J. (2015). Activating learned exemplars in children impairs memory for related exemplars in visual long-term memory. Visual Cognition, 23, 643-558. https://doi.org/10.1080/ 13506285.2015.1064052

Maxcey, A. M., Bostic, J., \& Maldonado, T. (2016). Recognition practice results in a generalizable skill in older adults: Decreased intrusion errors to novel objects belonging to practiced categories. Applied Cognitive Psychology, 30, 643-649. https://doi.org/10.1002/acp. 3236

Maxcey, A. M., Glenn, H., \& Stansberry, E. (2018). Recognition-induced forgetting does not occur for temporally grouped objects unless they are semantically related. Psychonomic Bulletin \& Review, 25, 10871103. https://doi.org/10.3758/s13423-017-1302-z

Maxcey, A. M., Janakiefski, L., Megla, E., Smerdell, M., \& Stallkamp, S. (2019). Modality-specific forgetting. Psychonomic Bulletin \& Review, 26, 622-633. https://doi.org/10.3758/s13423-019-01584-y 
Maxcey, A. M., McCann, M., \& Stallkamp, S. (2019). Recognition-induced forgetting is caused by episodic, not semantic, memory retrieval tasks. Manuscript under review.

Maxcey, A. M., \& Woodman, G. F. (2014). Forgetting induced by recognition of visual images. Visual Cognition, 22, 789-808.

McKoon, G., Ratcliff, R., \& Dell, G. S. (1986). A critical evaluation of the semantic-episodic distinction. Journal of Experimental Psychology: Learning, Memory, and Cognition, 12, 295-306. https://doi.org/10.1037//0278-7393.12.2.295

Melton, A. W. (1963). Implication of short-term memory for a general theory of memory. Journal of Verbal Learning and Verbal Behavior, 2, 1-21. https://doi.org/10.1016/S0022-5371(63)80063-8

Mirman, D., Landrigan, J.-F., \& Britt, A. E. (2017). Taxonomic and thematic semantic systems. Psychological Bulletin, 143, 499-520. https://doi.org/10.1037/bul0000092

Morey, R. D. (2008). Confidence intervals from normalized data: A correction to Cousineau (2005). Tutorials in Quantitative Methods for Psychology, 4, 61-64. https://doi.org/10.20982/tqmp.04.2.p061

Murayama, K., Miyatsu, T., Buchli, D., \& Storm, B. C. (2014). Forgetting as a consequence of retrieval: A meta-analytic review of retrievalinduced forgetting. Psychological Bulletin, 140, 1383-1409. https:// doi.org/10.1037/a0037505

Norman, K. A., Newman, E. L., \& Detre, G. (2007). A neural network model of retrieval-induced forgetting. Psychological Review, 114, 887-953. https://doi.org/10.1037/0033-295X.114.4.887

Ochsner, K. N., Chiu, C.-Y. P., \& Schacter, D. L. (1994). Varieties of priming. Current Opinion in Neurobiology, 4, 189-194.

Olson, I. R., \& Chun, M. M. (2001). Temporal contextual cueing of visual attention. Journal of Experimental Psychology: Learning, Memory, and Cognition, 27, 1299-1313. https://doi.org/10.1037/0278-7393. 27.5.1299

Raaijmakers, J. G. W., \& Jakab, E. (2013a). Is forgetting caused by inhibition? Current Directions in Psychological Science, 22, 205209.

Raaijmakers, J. G. W., \& Jakab, E. (2013b). Rethinking inhibition theory: On the problematic status of the inhibition theory for forgetting. Journal of Memory and Language, 68, 98-122. https://doi.org/10. 1016/j.jml.2012.10.002

Rakison, D. H., \& Oakes, L. M. (2003). Early category and concept development: Making sense of the blooming, buzzing confusion. New York, NY: Oxford University Press.

Rouder, J. N., Speckman, P. L., Sun, D., Morey, R. D., \& Iverson, G. (2009). Bayesian $t$ tests for accepting and rejecting the null hypothesis. Psychonomic Bulletin \& Review, 16, 225-237. https://doi.org/ 10.3758/PBR.16.2.225
Rugo, K. F., Tamler, K. N., Woodman, G. F., \& Maxcey, A. M. (2017). Recognition-induced forgetting of faces in visual long-term memory. Attention, Perception, \& Psychophysics, 79, 1878-1885. https:// doi.org/10.3758/s13414-017-1419-1

Schneider, W., Eschman, A., \& Zuccolotto, A. (2012). E-Prime reference guide. Pittsburgh, PA: Psychology Software Tools, Inc.

Sloutsky, V. M. (2010). From perceptual categories to concepts: What develops? Cognitive Science, 34, 1244-1286. https://doi.org/10. 1111/j.1551-6709.2010.01129.x

Storm, B. C., \& Levy, B. J. (2012). A progress report on the inhibitory account of retrieval-induced forgetting. Memory \& Cognition, 40, 827-843. https://doi.org/10.3758/s13421-012-0211-7

Taylor, J. R. (2003). Linguistic categorization. New York, NY: Oxford University Press.

Tulving, E. (1972). Episodic and semantic memory. In E. Tulving \& W. Donaldson (Eds.), Organization of memory (pp. 381-403). New York, NY: Academic Press.

Tulving, E., \& Schacter, D. L. (1990). Priming and human memory systems. Science, 247, 301-306. https://doi.org/10.1126/science. 2296719

Turk-Browne, N. B., Jungé, J. A., \& Scholl, B. J. (2005). The automaticity of visual statistical learning. Journal of Experimental Psychology: General, 134, 552-564. https://doi.org/10.1037/00963445.134.4.552

Valcke, M. (2002). Cognitive load: Updating the theory? Learning and Instruction, 12, 147-154.

Zentall, T. R., Wasserman, E. A., \& Urcuioli, P. J. (2014). Associative concept learning in animals. Journal of the Experimental Analysis of Behavior, 101, 130-151.

Open Practices Statement The stimuli and data are available on the Open Science Framework, at https://osf.io/5s96y/?view_only= e1801f074078415f90e0e3e536b33b5f. Experiment 2 is available to try online at https://maxceylab.github.io/expts/compelling scenes/5.html, and Experiment 3 is available to try online at https://maxceylab.github. io/expts/compelling scenes/2point5.html.

Publisher's note Springer Nature remains neutral with regard to jurisdictional claims in published maps and institutional affiliations. 